(C) 2016 IEEE. Personal use of this material is permitted. Permission from IEEE must be obtained for all other uses, in any current or future media, including reprinting/republishing this material for advertising or promotional purposes, creating new collective works, for resale or redistribution to servers or lists, or reuse of any copyrighted component of this work in other works. 


\title{
Improved Quality-Based Channel State Feedback Scheme in Multicast Systems with Greedy Scheduling
}

\author{
Hang Li, Xiaojing Huang, and Y. Jay Guo \\ University of Technology, Sydney \\ Email: \{Hang.Li, Xiaojing.Huang, Jay.Guo\}@uts.edu.au
}

\begin{abstract}
This paper proposes an Improved Quality-Based Channel State Feedback (IQCF) scheme in multicast systems with greedy scheduling for multiple multicast groups. The proposed scheme outperforms the Quality-Based Channel State Feedback (QCF) scheme in the literature by selecting a scheduling outage group for data transmission when scheduling outage happens. We also analyze its performance in terms of average capacity and outage probability, and present a closed-form system average capacity expression over Rayleigh fading channels. The numerically evaluated analytical results are verified by simulations.

Index Terms-Multicast systems, scheduling outage groups, system average capacity, outage probability, normalized average feedback load.
\end{abstract}

\section{INTRODUCTION}

To achieve higher spectral and power efficiencies, wireless multicast transmission has been widely adopted in $4 \mathrm{G}$ wireless communications standards (e.g., 3GPP LTE [1] and IEEE 802.16 [2]) as Evolved Multimedia Broadcast and Multicast Services (eMBMS). Taking advantage of multicast to simultaneously transmit the same message via a single point-tomultipoint link instead of many point-to-point (unicast) links, the available bandwidth in the networks can be efficiently utilized. Recently, multicast has been investigated and considered as one attractive solution in the emerging $5 \mathrm{G}$ wireless networks [3], [4] to improve the network throughput, energy efficiency and reliability.

Traditionally, in a wireless unicast system with greedy scheduling, the base station (BS) needs to select the mobile stations (MS) with the best channel quality for data transmission to maximize system capacity. Therefore, each MS is required to periodically feed back its channel state information (CSI) to the BS, which results in huge system feedback overhead. To reduce the feedback overhead, a Quality-Based Channel State Feedback (QCF) scheme [5] was proposed for wireless unicast systems with greedy scheduling, by which only the MSs whose instantaneous received signal to noise ratios (SNRs) are higher than a predetermined feedback threshold feed back their CSI to the BS, and the BS selects the MS with the best channel quality among the MSs with feedback for data transmission. Motivated by the QCF in unicast systems [5], a threshold based feedback scheme [6] was proposed for an orthogonal random beamforming based multicast system, by

This research was supported under Australian Research Council's Discovery Projects funding scheme (project number DP160101693).

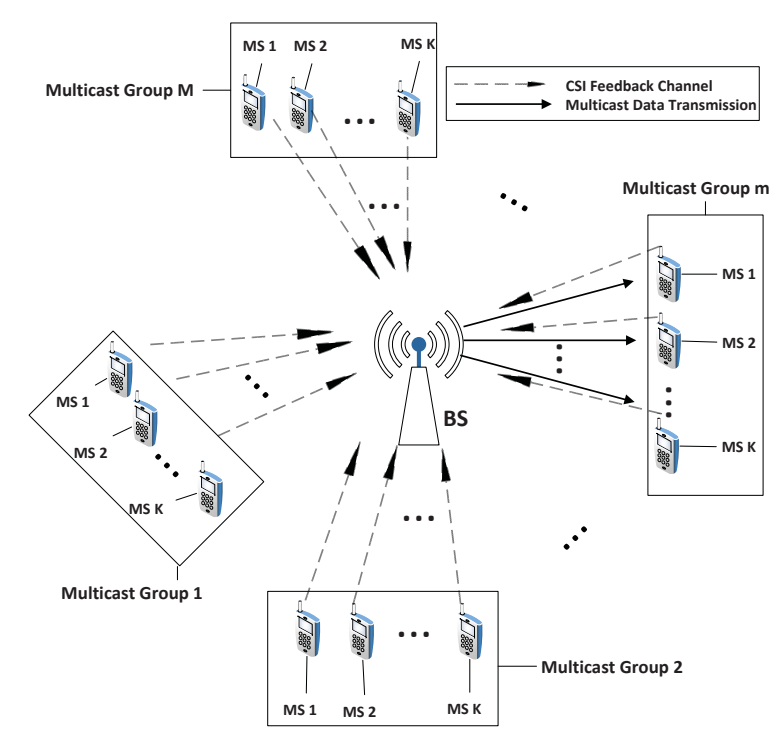

Fig. 1. A single-cell interference-free multicast network consisting of a BS and $M$ multicast groups, each with $K$ active MSs, where the multicast group $m$ is selected for data transmission.

which the MSs determine individually how to feed back their CSI with different decision rules. This scheme incurs a tolerable rate loss. In [7], a QCF scheme for multicast systems with greedy scheduling was proposed to reduce the feedback load with a negligible system performance loss, by which only the MSs whose instantaneous received SNRs are lower than a predetermined feedback threshold feed back their CSI to the BS. The reason is that the worst channel quality between the BS and the MSs in a multicast group is needed to determine the achievable capacity for that group. However, this scheme only considers the CSI of the scheduling non-outage groups, defined as the groups with feedback MSs in scheduling, but does not make use of the channel conditions of any scheduling outage group, defined as the group without any feedback MS. When there is scheduling outage, i.e., at least one muticast group has SNR higher than the feedback threshold, the BS can select the multicast group with the maximum capacity only among all scheduling non-outage groups. When no multicast group has SNR lower than the feedback threshold, the BS can not schedule any mutilcast group for data transmission.

In this paper, we propose an Improved Quality-Based Chan- 
nel State Feedback (IQCF) scheme, where the scheduling outage groups are selected in multicast transmission scheduling to improve system performance. When the scheduling outage occurs, the BS randomly schedules data transmission to a multicast group among all scheduling outage groups. Because the scheduling outage groups' channel qualities ${ }^{1}$ are better than the predetermined feedback threshold, but the scheduling nonoutage groups' channel qualities are worse than the predetermined feedback threshold, selecting a scheduling outage group will achieve higher capacity gain. Especially, with very limited feedback overhead (i.e., when very low feedback threshold is used), the scheduling outage occurs more frequently and hence the proposed IQCF scheme will perform even better. In the following sections, we first describe the system model and assumptions, then analyze the performance of the proposed IQCF in terms of average capacity and outage probability, and particularly present a closed-form system average capacity expression over Rayleigh fading channels.

\section{System Model}

\section{A. Downlink Transmission}

As shown in Fig. 1, we consider a downlink single-cell interference-free multicast network consisting of a BS and $M$ multicast groups, each with $K$ active MSs. Prior to scheduling data transmission, all active MSs' identity information has been allocated and recorded by the BS through a setup process, which can be carried out periodically (e.g., every one hour) and/or when a new MS or multicast group is registered in the network. In each scheduling, the BS only serves one selected multicast group for data transmission during any given slot. We make the following assumptions: (1) All active MSs are ideally synchronized to the BS. (2) The data for each multicast group are infinitely backlogged, therefore the queueing dynamics is not considered. (3) The channel is frequency flat fading, blockwise time invariant for each slot, and changes independently from slot to slot. (4) The channel gains $\left\{\left|h_{m, k}\right|^{2}\right\}$ from the BS to MS $k$ in group $m$ are independent and identically distributed (i.i.d.) random variables. Therefore, the instantaneous received $\operatorname{SNR}\left\{\gamma_{m, k}\right\}=\left\{\rho\left|h_{m, k}\right|^{2}\right\}$ are also i.i.d. random variables, where $\rho$ is the ratio of the BS's transmit power to the noise power at the receiver.

\section{B. Uplink Feedback Scheduling}

The total scheduling process for the IQCF can be described as follows. The BS first sends a multicast message ${ }^{2}$ to all multicast group MSs. Then, all active MSs synchronize with the BS and estimate their channel gains using the multicast message. Only the MSs, whose instantaneous received SNRs are lower than a predetermined feedback threshold $\eta$, feed back their CSI to the BS. We define $\gamma_{m}=\min _{k=1, \ldots, K}\left\{\gamma_{m, k}\right\}$ as the received SNR of group $m$. The achievable capacity $C_{m}$ for

\footnotetext{
${ }^{1}$ The channel quality of a multicast group is dominated by the worst channel quality among the MSs in that group.

${ }^{2}$ The multicast message carries system configuration information (e.g., the multicast groups' identities and the feedback threshold value).
}

group $m$ is subject to the minimum capacity link between the BS and the MSs in that group, i.e., $C_{m}=\log _{2}\left(1+\gamma_{m}\right)$. When every multicast group has feedback MSs (i.e., $\gamma_{m}<\eta, \forall m=$ $1, \ldots, M)$, based on greedy scheduling, the BS selects the group with the maximum received SNR among all groups (i.e., $\left.\max _{m=1, \ldots, M}\left\{\gamma_{m}\right\}\right)$ to schedule data transmission. In this case, the achievable capacity is $\max _{m=1, \ldots, M}\left\{C_{m}\right\}$.

On the other hand, when there is at least one scheduling outage group (i.e., $\gamma_{m} \geq \eta, \exists m=1, \ldots, M$ ), the BS randomly selects one group among all scheduling outage groups to initiate data transmission in accordance with the feedback threshold $\eta$ as the channel state. We assume that the feedback CSI can be always received without delay and error. In this case, the achievable capacity is $\log _{2}(1+\eta)$.

The above proposed IQCF considers scheduling outage groups with received SNRs greater than those of the scheduling non-outage groups (i.e., the scheduling outage groups always have higher capacity), whereas the QCF only considers the scheduling non-outage groups and selects the multicast group with the maximum capacity among the scheduling non-outage groups. When scheduling outage happens for all multicast groups, the QCF has to give up data transmission, while the IQCF is able to continue scheduling at an appropriate rate. Therefore, the IQCF brings capacity gain due to taking into account the scheduling outage groups.

\section{PERformance AnAlysis}

\section{A. Average Capacity}

Let $F_{\gamma_{m, k}}(x)$ be the cumulative distribution function (cdf) of $\gamma_{m, k}$. Without loss of generality, $F_{\gamma_{m, k}}(x)$ denotes the cdf of received SNR for any MS in any group due to i.i.d. random variables. Therefore, the cdf of $\gamma_{m}$ is given by

$$
\begin{aligned}
F_{\gamma_{m}}(x) & =\operatorname{Pr}\left\{\gamma_{m} \leq x\right\}=1-\operatorname{Pr}\left\{\gamma_{m}>x\right\} \\
& =1-\prod_{k=1}^{K} \operatorname{Pr}\left\{\gamma_{m, k}>x\right\} \\
& =1-\left(1-F_{\gamma_{m, k}}(x)\right)^{K} .
\end{aligned}
$$

Let $\gamma=\max _{m=1, \ldots, M}\left\{\gamma_{m}\right\}$. The cdf of $\gamma, F_{\gamma}(x)$, is then given by

$$
F_{\gamma}(x)=\operatorname{Pr}\{\gamma \leq x\}=\prod_{m=1}^{M} F_{\gamma_{m}}(x)=\left(F_{\gamma_{m}}(x)\right)^{M} .
$$

In the following, we focus on the probabilities that are pertained to a particular multicast group (i.e., the $m$ th group). Let $P_{m, 1}$ denote the probability that $\gamma_{m}<\eta$ and group $m$ is selected, and $P_{m, 2}$ denote the probability that $\gamma_{m} \geq \eta$ and group $m$ is selected. The system average capacity can then be written by

$$
\begin{aligned}
\bar{C}= & M P_{m, 1} \int_{0}^{\eta} \log _{2}(1+x) \frac{d F_{\gamma}(x)}{F_{\gamma}(\eta)} \\
& +M P_{m, 2} \log _{2}(1+\eta),
\end{aligned}
$$

where $\frac{F_{\gamma}(x)}{F_{\gamma}(\eta)}$ is the conditional cdf of $\gamma$ given $0 \leq \gamma<\eta$. 
Since the MSs in all groups are assumed to have the same channel statistics, the groups are equally likely to be selected by the BS with probability $1 / M$. Thus, we have $P_{m, 1}+P_{m, 2}=$ $\frac{1}{M}$, or $P_{m, 1}=\frac{1}{M}-P_{m, 2} \cdot P_{m, 2}$ is then given by

$$
\begin{aligned}
P_{m, 2}= & \operatorname{Pr}\left\{\gamma_{m} \geq \eta\right\} \sum_{n=0}^{M-1}\left(\begin{array}{c}
M-1 \\
n
\end{array}\right) \prod_{k=1}^{n} \operatorname{Pr}\left\{\gamma_{i_{k}} \geq \eta\right\} \\
& \times \prod_{k=1}^{M-1-n} \operatorname{Pr}\left\{\gamma_{j_{k}}<\eta\right\} \times \frac{1}{n+1} \\
= & \sum_{n=0}^{M-1}\left(\begin{array}{c}
M-1 \\
n
\end{array}\right)\left(1-F_{\gamma_{m}}(\eta)\right)^{n+1} \\
& \times\left(F_{\gamma_{m}}(\eta)\right)^{M-1-n} \times \frac{1}{n+1} \\
= & \frac{1}{M}\left(1-F_{\gamma}(\eta)\right),
\end{aligned}
$$

where $i_{1}, i_{2}, \ldots, i_{n}$ denote the indices of the MSs that do not feed back their CSI to the BS, and $j_{1}, j_{2}, \ldots, j_{M-1-n}$ denote the indices of the MSs that do feed back their CSI to the BS.

Substituting (4) into (3) and using integration by parts, the system average capacity can be simplified as

$$
\begin{aligned}
\bar{C}= & \frac{1-M P_{m, 2}}{F_{\gamma}(\eta)}\left[\left.\log _{2}(1+x) F_{\gamma}(x)\right|_{0} ^{\eta}\right. \\
& \left.-\log _{2} e \int_{0}^{\eta} \frac{F_{\gamma}(x)}{x+1} d x\right]+M P_{m, 2} \log _{2}(1+\eta) \\
= & \log _{2}(1+\eta)-\log _{2} e \int_{0}^{\eta} \frac{F_{\gamma}(x)}{x+1} d x .
\end{aligned}
$$

\section{B. Outage Probability}

The outage probability is defined as the probability that the achievable capacity falls below the predetermined target capacity $R$. Therefore, in a multicast system, the outage probability $O_{p}$ of the IQCF based on greedy scheduling is expressed as

$$
\begin{aligned}
O_{p} & =\operatorname{Pr}\{C<R\} \\
& =\operatorname{Pr}\left\{M P_{m, 1} \log _{2}(1+x)+M P_{m, 2} \log _{2}(1+\eta)<R\right\} \\
& =\operatorname{Pr}\left\{x<2^{A}-1\right\},
\end{aligned}
$$

where $A=\left[R-\left(1-F_{\gamma}(\eta)\right) \log _{2}(1+\eta)\right] / F_{\gamma}(\eta)$. As a result, (6) can be simplified as

$$
O_{p}= \begin{cases}1, & A>\log _{2}(1+\eta) \\ F_{\gamma}\left(2^{A}-1\right) / F_{\gamma}(\eta), & 0<A \leq \log _{2}(1+\eta) \\ 0 . & A \leq 0\end{cases}
$$

\section{Normalized Average Feedback Load}

To quantify the feedback load reduction by using the IQCF instead of the full CSI feedback (FCF) scheme [7], we define the normalized average feedback load $\bar{F}$ as the ratio of the IQCF average feedback load to the FCF load. The IQCF average feedback load $F_{I}$ is given by

$$
\begin{aligned}
F_{I} & =M \sum_{n=0}^{K} n\left(\begin{array}{c}
K \\
n
\end{array}\right)\left(F_{\gamma_{m, k}}(\eta)\right)^{n}\left(1-F_{\gamma_{m, k}}(\eta)\right)^{K-n} \\
& =M K F_{\gamma_{m, k}}(\eta)
\end{aligned}
$$

and the FCF load is $M K$. Therefore, the normalized average feedback load $\bar{F}=F_{\gamma_{m, k}}(\eta)$.

\section{Rayleigh Fading Channels}

We assume that all MSs' channels follow i.i.d. Rayleigh fading, and the average channel gains are normalized (i.e., $\left.E\left\{\left|h_{m, k}\right|^{2}\right\}=1\right)$. Therefore, the instantaneous received SNRs $\left\{\gamma_{m, k}\right\}$ follow exponential distribution with the cdf given by

$$
F_{\gamma_{m, k}}(x)=1-e^{-x / \rho}, \quad x \geq 0 .
$$

Substituting (9) into (1) and (2), we have

$$
F_{\gamma}(x)=\left(1-e^{-K x / \rho}\right)^{M}, \quad x \geq 0 .
$$

By substituting (10) into (5) and using the binomial expansion, the system average capacity $\bar{C}$ in closed-form for Rayleigh fading channels is given by

$$
\begin{aligned}
\bar{C}= & -\log _{2} e \sum_{n=1}^{M}\left(\begin{array}{c}
M \\
n
\end{array}\right)(-1)^{n} \int_{0}^{\eta} \frac{e^{-n K x / \rho} d x}{x+1} \\
= & \log _{2} e \sum_{n=1}^{M}\left(\begin{array}{c}
M \\
n
\end{array}\right)(-1)^{n} e^{n K / \rho} \\
& \times\left[\operatorname{Ei}\left(-\frac{n K}{\rho}\right)-\operatorname{Ei}\left(-\frac{n K(1+\eta)}{\rho}\right)\right],
\end{aligned}
$$

where the Eq. (3.352.1) in [8] is used as follows:

For $u \geq 0$ and $|\arg \beta|<\pi$,

$$
\int_{0}^{u} \frac{e^{-\mu x} d x}{x+\beta}=e^{\mu \beta}[\operatorname{Ei}(-\mu u-\mu \beta)-\operatorname{Ei}(-\mu \beta)],
$$

and $\operatorname{Ei}(\cdot)$ is the exponential integral function defined by $\operatorname{Ei}(x)=$ $\int_{-\infty}^{x} e^{t} / t d t$

The outage probability $O_{p}$ in Rayleigh fading channels can be easily obtained by substituting (10) into (7), and the normalized average feedback load $\bar{F}=1-e^{-\eta / \rho}$. It is noteworthy that, for Rayleigh fading channels, $\bar{F}$ is only dominated by $\eta$ and $\rho$, but not by $M$ or $K$. If the feeback load is limited, we may choose the optimum threshold $\eta$ on the premise of a certain normalized average feedback load. This can be achieved as $\eta=-\rho \ln (1-\bar{F})$.

\section{NUMERICAL RESUlts}

In this section, we present the numerical results for the proposed IQCF with the greedy scheduling over Rayleigh fading channels with $\rho=10 \mathrm{~dB}$, which are verified through Matlab simulations. As shown in Figs. 2 and 3, the numerical results with solid lines match the simulation results with markers. We also compare these results with those of QCF in [7].

Fig. 2 shows system average capacity $\bar{C}$ versus the normalized average feedback load $\bar{F}$ for the IQCF and QCF. As $\bar{F}$ increases, i.e., the feedback threshold $\eta$ increases, the system average capacity $\bar{C}$ increases. When $\bar{F}$ approaches to $1, \bar{C}$ for IQCF reaches that of FCF. The IQCF has less feedback load than QCF when they have the same system average capacity. In the scenario of $M=3$ and $K=6$, when there is a performance 


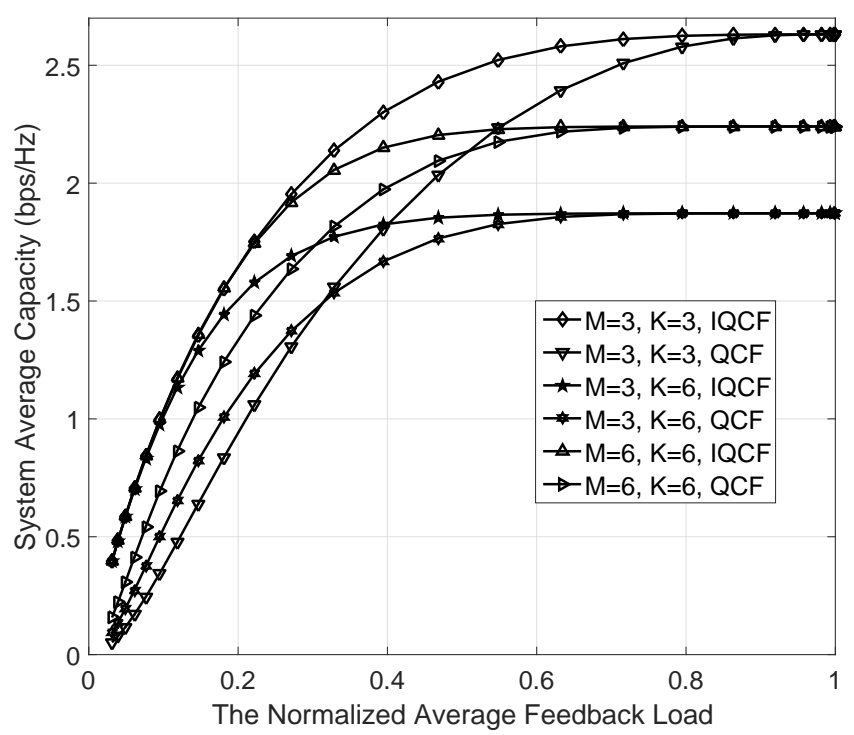

Fig. 2. System average capacity $\bar{C}$ versus the normalized average feedback load $\bar{F}$.

loss of about $10 \%$ compared with that of FCF, the IQCF only uses $26 \%$ feedback load, while the QCF uses $40 \%$. Likewise, the IQCF offers better system average capacity than the QCF with the same limited amount of feedback load. For example, the IQCF achieves about $46 \%$ capacity gain of the QCF with $M=3$ and $K=3$, when $\bar{F}$ is limited to $30 \%$.

As also shown in Fig. 2, the gain gradually decreases with the increase of $\bar{F}$. The reason is as follows. When the amount of the feedback load is limited (i.e., the comparatively small $\eta$ is used), the probability that there exist scheduling outage groups is relatively high, and the system average capacity attributed to scheduling outage groups for the IQCF always outperforms that of the QCF's scheduling non-outage groups. As $\bar{F}$ increases, the probability that there exist scheduling outage groups becomes small, and the gain achieved by scheduling outage groups is reduced. It is observed that the capacity performance is degraded with the increase of the number of MSs in a group when the number of groups is fixed, becasuse the achievable capacity for a multicast group is dominated by the MS with the lowest received SNR among all MSs. On the other hand, the capacity performance is improved with the increase of the number of groups when the number of MSs in a group is fixed, which is due to the multicast groups' diversity gain.

Fig. 3 shows the outage probability $O_{p}$ versus the feedback threshold $\eta$ for the IQCF and QCF with $R=1.1,1.5$ and 2 respectively. It can be seen that when the feedback threshold $\eta$ exceeds a certain value, the outage performance of the IQCF is far better than that of the QCF along with the decrease of the predetermined target capacity $R$. As $\eta$ approaches to $10 \mathrm{~dB}$, the IQCF and QCF achieve the same outage performance. Particularly, the outage probability of the IQCF with $R=1.1$ drops dramatically as $\eta$ increases from $0.6 \mathrm{~dB}$ to $2.6 \mathrm{~dB}$, because the scheduling outage groups play a dominant role in system

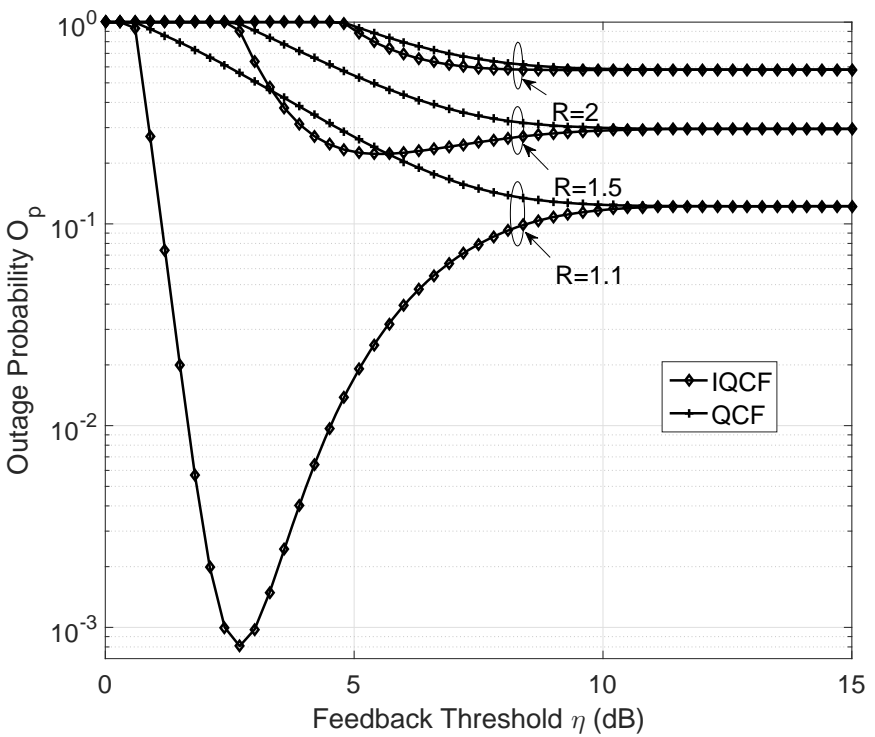

Fig. 3. Outage probability $O_{p}$ versus feedback threshold $\eta$.

capacity compared with scheduling non-outage groups as $\eta$ increases. However, as $\eta$ increases from $2.6 \mathrm{~dB}$, the outage probability increases gradually to a constant due to the decrease of the capacity achieved by scheduling outage groups.

\section{Conclusion}

In this paper, we propose the IQCF scheme, by which the scheduling outage groups are taken into account in scheduling to improve the system performance. Then, we analyze its performance over general channels in terms of average capacity and outage probability, and present a closed-form system average capacity expression over Rayleigh fading channels. Numerical and simulation results show that, compared with QCF, the IQCF saves more feedback load with an acceptable average capacity loss. Meanwhile it achieves higher average capacity gain especially when the amount of feedback load is very limited.

\section{REFERENCES}

[1] Multimedia Broadcast/Multicast Service (MBMS); Stage 1 (Release 11). 3GPP TS 22.146 Version 11.1.0, May 2013.

[2] The Draft IEEE $802.16 \mathrm{~m}$ System Description Document, Feb. 2009, available: http://wirelessman.org/tgm/docs/80216m-08_003r7.zip.

[3] K. Poularakis, G. Iosifidis, V. Sourlas, and L. Tassiulas, "Exploiting caching and multicast for 5G wireless networks," IEEE Trans. Wireless Commun., to be published in 2016.

[4] M. Ji, A. M. Tulino, J. Llorca, and G. Caire, "Caching and coded multicasting: Multiple groupcast index coding," in Proc. IEEE Global Conf. Signal Inf. Process., Dec. 2014, pp. 881-885.

[5] D. Kim and I.-H. Lee, "On capacity of quality-based channel-state reporting in mobile systems with greedy transmission scheduling, IEEE Trans. Commun., vol. 54, no. 6, pp. 975-979, Jun. 2006.

[6] Y.-Y. Chang, W.-S. Liao, J.-H. Li, and H.-J. Su, "Channel feedback reduction for wireless multimedia broadcast multicast service systems," in Proc. IEEE ICC., Jun. 2015, pp. 4443-4447.

[7] I.-H. Lee and S.-C. Kwon, "Performance analysis of quality-based channel state feedback scheme for wireless multicast systems with greedy scheduling," IEEE Commun. Lett., vol. 19, no. 8, pp. 1430-1433, Aug. 2015.

[8] I. S. Gradshteyn and I. M. Ryzhik, Table of Integrals, Series, and Products, 7th ed. San Diego, CA, USA: Academic, 2007. 